\title{
Thalamic gap junctions control local neuronal synchrony and influence macroscopic oscillation amplitude during EEG alpha rhythms
}

\section{Stuart W. Hughes ${ }^{1 *}$, Magor L. Lőrincz ${ }^{1 \dagger}$, Kate Blethyn ${ }^{1}$, Katalin A. Kékesi ${ }^{2,3}$, Gábor Juhász ${ }^{3}$, Mark Turmaine $^{4}$, John G. Parnavelas ${ }^{4}$ and Vincenzo Crunelli ${ }^{1}$}

1 Neuroscience Division, School of Biosciences, Cardiff University, Cardiff, UK

2 Department of Physiology and Neurobiology, Eötvös Loránd University, Budapest, Hungary

${ }^{3}$ Laboratory of Proteomics, Institute of Biology, Eötvös Loránd University, Budapest, Hungary

4 Department of Cell and Development Biology, University College London, London, UK

\section{Edited by:}

Ole Jensen, Radboud University,

Netherlands

Reviewed by:

Stephanie R. Jones, Harvard Medical

School, USA

Diego Contreras, University of

Pennsylvania School of Medicine, USA

*Correspondence:

Stuart W. Hughes, Lilly UK, Erl Wood

Manor, Windlesham, Surrey GU2O

6PH, UK.

e-mail: hughes_stuart@lilly.com

${ }^{+}$Current Address:

Magor L. Lörincz, Instituto Gulbenkian

de Ciênca, Rue de Quinta Grande, 6,

2780-156 Oeiras, Portugal.
Although EEG alpha $(\alpha ; 8-13 \mathrm{~Hz})$ rhythms are often considered to reflect an "idling" brain state, numerous studies indicate that they are also related to many aspects of perception. Recently, we outlined a potential cellular substrate by which such aspects of perception might be linked to basic $\alpha$ rhythm mechanisms. This scheme relies on a specialized subset of rhythmically bursting thalamocortical (TC) neurons (high-threshold bursting cells) in the lateral geniculate nucleus (LGN) which are interconnected by gap junctions (GJs). By engaging GABAergic interneurons, that in turn inhibit conventional relay-mode TC neurons, these cells can lead to an effective temporal framing of thalamic relay-mode output. Although the role of GJs is pivotal in this scheme, evidence for their involvement in thalamic $\alpha$ rhythms has thus far mainly derived from experiments in in vitro slice preparations. In addition, direct anatomical evidence of neuronal GJs in the LGN is currently lacking. To address the first of these issues we tested the effects

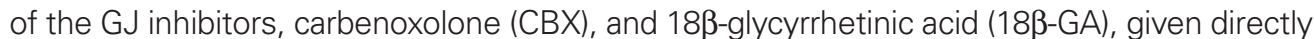
to the LGN via reverse microdialysis, on spontaneous LGN and EEG $\alpha$ rhythms in behaving cats. We also examined the effect of CBX on $\alpha$ rhythm-related LGN unit activity. Indicative of a role for thalamic GJs in these activities, 18ß-GA and CBX reversibly suppressed both LGN and EEG $\alpha$ rhythms, with CBX also decreasing neuronal synchrony. To address the second point, we used electron microscopy to obtain definitive ultrastructural evidence for the presence of GJs between neurons in the cat LGN. As interneurons show no phenotypic evidence of GJ coupling (i.e., dye-coupling and spikelets) we conclude that these GJs must belong to TC neurons. The potential significance of these findings for relating macroscopic changes in $\alpha$ rhythms to basic cellular processes is discussed.

Keywords: EEG, gap junctions, electrical synapse, alpha rhythms, acetylcholine, metabotropic glutamate receptor

\section{INTRODUCTION}

Although the customary view of EEG $\alpha(8-13 \mathrm{~Hz})$ rhythms is that they are a definitive marker of quiet or relaxed wakefulness, there is a large body of evidence indicating that they are also closely linked to several important aspects of perception (VanRullen and Koch, 2003; Fingelkurts and Fingelkurts, 2006; Mathewson et al., 2009). For example, it has been known for some time that both reaction time (Surwillo, 1961) and the maximal inter-stimulus interval for perceived simultaneity (Kristofferson, 1967) are tightly correlated with $\alpha$ rhythm frequency. More recently, there have been reports suggesting that the ability to accurately perceive the temporal order of specific events (Varela et al., 1981), or even to perceive them at all (Nunn and Osselton, 1974; Busch et al., 2009; Mathewson et al., 2009), might be dependent on the particular phase of the $\alpha$ rhythm at which they take place. Of course, the idea that the phase of $\alpha$ rhythms influences perception in this way is not new and has been a recurring theme of $\alpha$ rhythm research since the discovery of the dominant occipital rhythm as the first periodic signal in the EEG over 80 years ago (Berger, 1929). The central tenet of these studies is that $\alpha$ rhythms provide "excitability cycles" that act to temporally frame or gate perceptual events (Bartley, 1940; Lindsley, 1952; Lansing, 1957; Wiener, 1985; Crick and Koch, 2003; VanRullen and Koch, 2003; Fingelkurts and Fingelkurts, 2006; Mathewson et al., 2009). Ultimately, such "excitability cycles" are considered to be a potential basis for explaining discrete perceptual processing in the brain, an idea whereby perception is considered to occur in discrete snapshots or processing epochs lasting around $70-100 \mathrm{~ms}$ (Stroud, 1955; Efron, 1970), and which itself can be traced back to the work of William James over a century ago (James, 1890).

Somewhat surprisingly, although the idea that $\alpha$ rhythms provide a temporal framework for perception has often been discussed and debated, until recently, evidence at the cellular level of a link between $\alpha$ rhythms and the activity of neurons thought to be involved in perceptual processing was lacking. One of the main reasons for this was a lack of understanding of $\alpha$ rhythm mechanisms at the neuronal level. To redress this, we recently undertook a series of studies aimed at uncovering the basic cellular mechanisms that lead to $\alpha$ rhythms in the cat visual thalamus, i.e., the lateral 
geniculate nucleus (LGN). Our specific interest in this structure was fueled by earlier work in both cats (Chatila et al., 1992, 1993; Rougeul-Buser and Buser, 1997) and dogs (Lopes da Silva et al., 1973a,b) demonstrating that it plays a central role in driving cortical $\alpha$ rhythms that are essentially equivalent to those observed in humans. In carrying out this work, we identified a specialized subset of thalamocortical (TC) neurons which exhibit intrinsic rhythmic burst activity in the $\alpha$ frequency band (termed high-threshold, HT, bursting) and which are synchronized by electrical synapses or gap junctions (GJs) to form a coordinated $\alpha$ rhythm pacemaker (Hughes et al., 2004; Hughes and Crunelli, 2005; Lorincz et al., 2008, $2009 b)$. We further showed that by exciting local circuit interneurons, which in turn inhibit TC neurons that fire in a conventional single-spike or so-called relay-mode, this group of cells could generate an effective $\alpha$ rhythm-derived temporal framing of LGN relay-mode output (Lorincz et al., 2009b). Some LGN relay-mode TC neurons also appear to receive direct GJ-mediated connections from the HT bursting cell network, thereby further strengthening the link between thalamic relay-mode activity and local rhythmogenic machinery (Lorincz et al., 2008, 2009b). Because relay-mode firing in the LGN is the mechanism by which incoming visual signals are transmitted to the primary visual cortex, this type of firing is a necessary component in determining how visual information is ultimately perceived. Given this, the eventual periodic framing of relay-mode activity by oscillatory processes (i.e., GJ-coupled HT bursting cells) that are also responsible for generating $\alpha$ rhythms is therefore a possible route by which $\alpha$ rhythms may be linked to perception.

Arguably, the most fundamental component of the scheme outlined above for linking $\alpha$ rhythms to primitive perceptual processing is the presence of the basic rhythm-generating combination of HT bursting cells and GJ coupling. However, despite this, and although the existence of HT bursting TC neurons in vivo has been clearly documented (Hughes et al., 2004; Lorincz et al., 2009b), evidence for an involvement of thalamic GJs in controlling $\alpha$ rhythms has so far stemmed largely from experiments carried out in a reduced slice preparation of the LGN where the capacity to exhibit $\alpha$ rhythms is preserved (Hughes et al., 2004; Lorincz et al., $2008,2009 b)$. On top of this, even in in vitro experiments, direct and unequivocal evidence for the presence of neuronal GJs in the LGN is currently lacking. To address the first of these issues, we obtained simultaneous recordings of the occipital EEG, the LGN local field potential (LFP) and LGN unit activity during natural wakefulness in behaving cats and observed the effects of delivering the known GJ inhibitors, carbenoxolone (CBX), and $18 \beta$-glycyrrhetinic acid (18ß-GA; Davidson and Baumgarten, 1988), directly to the LGN via reverse microdialysis. Commensurate with a role for thalamic GJs in the generation of $\alpha$ activity, these agents suppressed both the density and power of LGN and EEG $\alpha$ rhythms. On the other hand, the glycyrrhetinic acid derivative that is inactive as a GJ inhibitor, glycyrrhizic acid (GZA), had no effect. CBX also decreased local neuronal synchrony during $\alpha$ rhythms. To address the second issue we obtained ultrathin sections from the LGN of adult cats and showed, using both conventional and freeze-fracture electron microscopy (EM), the unequivocal presence of neuronal GJs. Furthermore, because we were only able to identify phenotypic evidence of GJs between TC neurons, we conclude that it is these cells, rather than local circuit interneurons, to which the detected GJs belong. The implications of these results for relating the largescale dynamics of $\alpha$ rhythms to basic cellular processes is discussed.

\section{MATERIALS AND METHODS}

All in vivo and in vitro experiments were carried out in accordance with the guidelines of the local ethical committees, the UK Animals (Scientific Procedure) Act, 1986 and the Hungarian Act of Animal Care and Experimentation (1998. XXVIII. Section 243/1998), which conforms to the European Community regulations (86/609/). All efforts were made to minimize the suffering and number of animal used in each experiment.

\section{SURGERY AND IMPLANTATION FOR IN VIVO RECORDINGS}

Surgery for chronic implantation was carried out as described previously (Hughes et al., 2004; Lorincz et al., 2009b). Briefly, adult cats $(3.2-4.5 \mathrm{~kg})$ were anesthetized with $40 \mathrm{mg} / \mathrm{kg}$ Nembutal and placed into a stereotaxic frame (David Kopf 900 series, David Kopf Instruments, Tujunga, USA). Stainless steel screws $(0.8 \mathrm{~mm})$ were implanted above the occipital and parietal cortices for EEG recording. Bilateral $3 \mathrm{~mm}$ holes were drilled into the bone for implanting electrode arrays (see below) at coordinates A: 7.2, L: 9.5-10, V: $+6 \mathrm{~mm}$ (Berman and Jones, 1982). These are located in lamina A of the LGN and correspond to an area which we have previously identified as being important for $\alpha$ rhythm generation (Hughes et al., 2004; Hughes and Crunelli, 2005; Lorincz et al., 2008, 2009b). Cats were allowed to recover from the implantation for at least 7 days before recording commenced. For recording extracellular unit activity and LFPs from the LGN, cats were chronically implanted with microelectrodes. Two custom made bundles consisting of 8 or 16 Teflon-insulted $25 \mu \mathrm{m} \mathrm{Pt/Ir} \mathrm{wires} \mathrm{and} \mathrm{one} 80 \mu \mathrm{m} \mathrm{Ni} / \mathrm{Cr}$ wire (150-300 k $\Omega$ impedance at $1 \mathrm{kHz}$ ) were attached to the outer walls of a 22-Gage polyethylene guide cannula such that the tips of the electrodes were $10 \mathrm{~mm}$ below the base of the cannula. The guide cannula was then attached to the piston of a cylinder-piston-type microdrive (Hughes et al., 2004; Lorincz et al., 2009b). Local thalamic drug application was achieved using reverse microdialysis. Custom made microdialysis probes ( $4 \mathrm{~mm}$ length, hollow fiber, $50 \mathrm{kDa}$ molecular weight cut-off, $200 \mu \mathrm{m}$ outer diameter) were inserted into 27-Gage stainless steel tubing. This stainless steel tubing was then inserted into the polyethylene guide cannula. Once inserted, the microdialysis probe was fixed with two screws to the top of the metal cylinder to prevent any further movement. The turning of the piston thread, therefore, only caused the electrode bundles to slide, while the probe was maintained in a constant position.

During experiments, the animals were allowed to move freely in a sound-proofed, electrically shielded, and behaviorally enriched environment. Stages of vigilance were determined from a combination of EEG and behavioral criteria as fully described previously (Hughes et al., 2004; Lorincz et al., 2009b). During episodes of $\alpha$ activity cats were in a state of quiet or relaxed wakefulness and generally assumed the "sphinx" position. As such, occurrences of $\alpha$ waves did not correspond to any overt behavior and we did not set out to correlate $\alpha$ activity with any specific behavioral task or context. Rather, periods of activity were chosen according to the amount of $\alpha$ activity exhibited in the LFP (confirmed 
post hoc through examination of corresponding power spectra), specifically defined as periods when $\alpha$ waves were at least twice the amplitude of normal baseline activity (defined as activity corresponding to wakefulness when $\alpha$ activity was not present; Hughes et al., 2004; Lorincz et al., 2009b). Accordingly, $\alpha$ rhythm density was defined as the percentage of time taken up in any given $60 \mathrm{~s}$ period by rhythmic $\alpha$ activity defined in such a way. Extracellular signals were amplified by a Supertech Multiamp amplifier (Supertech, Pécs, Hungary) and then digitally acquired with a CED 1401 (Cambridge Electronic Design Ltd., Cambridge, UK) for storage and offline analysis on a personal computer or were amplified and acquired by a 64 channel Plexon integrated recording system (Plexon Inc., Dallas, USA). Unless explicitly stated otherwise, field and unit signals were isolated by post hoc digital band-pass filtering at 2-20 and $0.3-10 \mathrm{kHz}$, respectively. All drugs effects were assessed after $1 \mathrm{~h}$ of administration whereas wash effects were evaluated $2 \mathrm{~h}$ after re-introduction of vehicle (i.e., artificial cerebrospinal fluid, ACSF). At the end of an experiment, animals were given a lethal injection of Nembutal. For all animals, electrode tracks and final tip positions were confirmed post mortem in histological sections.

\section{IN VIVODATA ANALYSIS AND NEURON CLASSIFICATION}

Recorded units were identified as TC neurons, as opposed to interneurons, on the basis that during NREM sleep or under ketamine/xylazine anesthesia they exhibited unequivocal lowthreshold $\mathrm{Ca}^{2+}$-potential (LTCP)-mediated bursts (Domich et al., 1986; Hughes et al., 2004; Lorincz et al., 2009b). An LTCP-mediated burst was defined as a group of two or more spikes exhibiting a first ISI of $\leq 6 \mathrm{~ms}$, subsequent intervals of $<15$ ms which progressively increased, and a preceding silent period of at least $200 \mathrm{~ms}$. HT bursts were defined as a group of two or more spikes exhibiting a preceding silent period of at least $70 \mathrm{~ms}$ and ISIs that were between 3 and $20 \mathrm{~ms}$ and which (for bursts of more than two spikes) did not systematically increase as the burst progressed. HT bursting neurons were also required to exhibit two clear peaks in their corresponding interspike interval histograms (corresponding to the inter- and intra-burst intervals). All TC neurons that did not exhibit HT bursts were classified as tonic firing/relay-mode neurons. The definitions of both HT and LTCP-mediated bursts were based on extensive in vitro intracellular recordings from the cat LGN (Pirchio et al., 1997; Hughes, et al., 2002b; Hughes et al., 2004). Cross-correlograms of simultaneously recorded units and power spectra were computed from 10 consecutive $\alpha$ rhythm epochs. Cell pairs were considered to be correlated if the corresponding cross-correlogram exhibited a central peak which exceeded the $95 \%$ confidence limit. To construct a spike-timing histogram, the times of at least 200 spikes (comprising groups of consecutive spikes from several consecutive $\alpha$ rhythm epochs) were determined relative to the nearest negative peaks of the LFP using custom written transform routines in SigmaPlot 9 (Systat, Hounslow, UK). These times were subsequently assigned a given phase between these peaks (i.e., between $0^{\circ}$ and $360^{\circ}$ ) and then binned at $36^{\circ}$. For clarity, and to afford a sense of rhythmicity, this data was repeated over at least one additional full cycle of the oscillation $\left(-360^{\circ}\right.$ to $\left.0^{\circ}\right)$ to produce the final plot (Hughes et al., 2004; Lorincz et al., 2008, 2009b). Unless otherwise stated all quantitative in vivo data are expressed as mean \pm SEM and statistical significance was assessed with either Student's $t$-test or an ANOVA test.

\section{IN VITRO SLICE PREPARATION AND MAINTENANCE AND IN VITRO ELECTROPHYSIOLOGY}

Young adult cats (1-1.5 kg) were deeply anesthetized with a mixture of $\mathrm{O}_{2}$ and $\mathrm{NO}_{2}(2: 1)$ and $2.5 \%$ halothane, a wide craniotomy performed and the brain removed. Sagittal slices of the LGN were prepared and maintained as described previously (Hughes et al., 2002a,b, 2004; Lorincz et al., 2008, 2009a,b). For recording, slices $(450-500 \mu \mathrm{m})$ were perfused with a warmed $\left(35 \pm 1^{\circ} \mathrm{C}\right)$ continuously oxygenated $\left(95 \% \mathrm{O}_{2}, 5 \% \mathrm{CO}_{2}\right)$ ACSF containing (mM): $\mathrm{NaCl}$ (134); $\mathrm{KCl}(2) ; \mathrm{KH}_{2} \mathrm{PO}_{4}(1.25) ; \mathrm{MgSO}_{4}(1) ; \mathrm{CaCl}_{2}(2) ; \mathrm{NaHCO}_{3}(16)$; glucose (10). Intracellular recordings, using the current clamp technique, were performed with standard-wall glass microelectrodes filled with $1 \mathrm{M}$ potassium acetate (KAc; resistance: $80-120 \mathrm{M} \Omega$ ), and in some cases $2 \%$ biocytin or neurobiotin, and connected to an Axoclamp-2A amplifier (Axon Instruments, Foster City, CA, USA) operating in bridge mode. As described previously neurons were recorded in the presence of either $100 \mu \mathrm{M}$ trans-ACPD or $50 \mathrm{mM}$ carbachol (Cch) to facilitate the detection of spontaneous spikelets and excitatory post-synaptic potentials (EPSPs; Hughes et al., 2002a, 2004; Lorincz et al., 2008, 2009a,b). For experiments involving the injection of either biocytin or neurobiotin, at the end of the recording session slices were left in the recording chamber for at least $1 \mathrm{~h}$ prior to being fixed and then processed as described previously (Williams et al., 1996). Fixed sections were re-sectioned at $100 \mu \mathrm{m}$ (Crunelli et al., 1987; Pirchio et al., 1997).

\section{IN VITRO DATA ANALYSIS AND NEURON IDENTIFICATION}

Impaled cells were identified as TC neurons using established criteria (Pirchio et al., 1997; Hughes et al., 2002a,b, 2004; Lorincz et al., 2008, 2009b). Interneurons were identified morphologically using well-recognized criteria (McCormick and Pape, 1988; Pape and McCormick, 1995; Williams et al., 1996; Cox et al., 2003) and electrophysiologically by a significantly shorter action potential half-width and smaller action potential after hyperpolarization (McCormick and Pape, 1988; Pape and McCormick, 1995; Williams et al., 1996; Lorincz et al., 2009b). Voltage and current records were digitally acquired and processed using pClamp 9 (Molecular Devices Corporation, Sunnyvale, CA, USA). Apparent input resistance was estimated from the responses to small (10-100 pA) pulses applied from a membrane potential of $-60 \mathrm{mV}$. Unless otherwise stated all quantitative in vitro data are expressed as mean \pm SEM and statistical significance was assessed with either Student's $t$-test or an ANOVA test.

\section{ELECTRON MICROSCOPY}

Two young adult cats were perfused with a fixative solution containing $1 \%$ paraformaldehyde and $1.25 \%$ glutaraldehyde in $0.1 \mathrm{M}$ phosphate buffer (PB; pH 7.2-7.4). Following perfusion, the animals were left intact for several hours before removing the thalamus from each brain hemisphere and placing in fixative solution for $2 \mathrm{~h}$. The lateral geniculate nuclei were then dissected and placed in $\mathrm{PB}$ before sectioning at $200 \mu \mathrm{m}$ in the coronal plane with a Vibratome. The tissue slices were subsequently postfixed in $2 \% \mathrm{OsO}_{4}$ in $\mathrm{PB}$ 
for $2 \mathrm{~h}$, rinsed in buffer, stained in aqueous $1 \%$ uranyl acetate for $1.5 \mathrm{~h}$, dehydrated in ethanol, and embedded in Araldite. Ultrathin sections were cut through the LGN, collected on 200-mesh grids and examined with a JEOL 1010 electron microscope.

For freeze-fracture EM, Vibratome-cut tissue slices were equilibrated with $12.5 \%$ and $25 \%$ glycerol in $\mathrm{PB}$ for $2 \mathrm{~h}$ and rapidly frozen in liquid Freon. Fracturing and replication were done in a Cressington CFE freeze-fracture apparatus at $-140^{\circ} \mathrm{C}$; the replicas were coated with carbon and platinum, cleaned by floating in sodium hypochloride, and examined with the electron microscope.

\section{SOURCES OF DRUGS}

DL-2-amino-5-phosphonovaleric acid (APV), 2,3-dioxo-6-nitro1,2,3,4-tetrahydrobenzo[f] quinoxaline-7-sulfonamide disodium salt (NBQX), and ( \pm )-1-aminocyclopentane-trans-1,3-dicarboxylic acid (trans-ACPD) were obtained from Tocris-Cookson (UK); carbamylcholine chloride (carbachol, Cch), carbenoxolone (CBX), 18 $\beta$-glycyrrhetinic acid (18 $\beta$-GA), and glycyrrhizic acid (GZA) were obtained from Sigma (UK). APV, Cch, NBQX, and trans-ACPD were dissolved directly in ACSF. 18 $\beta$-GA, CBX, and GZA were dissolved in DMSO and then added to ACSF such that the total final volume of DMSO did not exceed $0.1 \%$.

\section{RESULTS}

\section{ALPHA $(\alpha)$ RHYTHM EPISODES IN THE CAT DURING RELAXED WAKEFULNESS}

As described previously (Hughes et al., 2004; Lorincz et al., 2009b), during periods of quiet wakefulness, EEG recordings in freely moving cats obtained from the occipital cortex regularly exhibited periods of prominent $\alpha$ rhythms that occurred synchronously with rhythmic activity in LFP recordings from the LGN (mean frequency: $9.3 \pm 0.3 \mathrm{~Hz} ; n=30$ episodes; Figure 1A). Again, consistent with earlier reports, simultaneously acquired, single unit recordings from the LGN using multi-electrode, fine-wire bundles confirmed that the activity of TC neurons during $\alpha$ rhythms consisted of either HT bursting (Figure 1B1) or single-spike activity (i.e., tonic or relay-mode firing; Figure 1B2). For HT bursting cells, spikes typically occurred predominantly close to the negative $\alpha$ rhythm peak in the LFP whereas for tonic cells, spikes were inhibited close to either the $\alpha$ rhythm peak or trough (for a quantitative description and fuller explanation of these firing patterns see Lorincz et al., 2009b).

\section{THALAMIC DELIVERY OF GAP JUNCTION BLOCKERS DISRUPTS LGN AND EEG A RHYTHMS}

In order to assess the role of thalamic GJs in the generation of LGN and EEG $\alpha$ rhythms we examined the effects of carbenoxolone (CBX) and $18 \beta$-glycyrrhetinic acid (18 $\beta$-GA; both at $1 \mathrm{mM})$, delivered directly to the LGN via reverse microdialysis, on spontaneous oscillatory activity. These agents brought about a significant suppression of both LGN and EEG $\alpha$ rhythms (Figures 2A-D; Table 1). This was manifested as reduction in both $\alpha$ rhythm density and $\alpha$ rhythm power (Figures 2A-D; Table 1). Consistent with in vitro findings (cf. Figure 6 in Hughes et al., 2004 and Figure 1 in Lorincz et al., 2008), neither CBX nor 18 $\beta$-GA significantly affected LGN or EEG $\alpha$ rhythm frequency (Figures $2 \mathrm{E}, \mathrm{F}$; Table 1 ). In contrast to the suppressive effects of CBX and $18 \beta-$ GA, the glycyrrhetinic acid derivative that is inactive as a GJ blocker, glycyrrhizic acid (GZA, $1 \mathrm{mM}$ ), had no effect on either the density, power, or frequency of LGN and EEG $\alpha$ rhythms (Figures 2B,D,F; Table 1).

\section{SYNCHRONIZED RHYTHMIC FIRING OF TC NEURONS DURING $\alpha$ RHYTHMS IS REVERSIBLY SUPPRESSED BY LOCAL DELIVERY OF CARBENOXOLONE (CBX)}

During the course of this study we were able to simultaneously record several pairs of TC neurons that displayed synchronized firing during spontaneous $\alpha$ rhythm epochs. These comprised both HT bursting/HT bursting TC neurons pairs ( $n=5$; Figure 3A, upper panel) and HT bursting/relay-mode TC neuron pairs $(n=7$; Figure 3A, lower panel). To test whether GJs are operational in synchronizing this firing we again applied the GJ blocker, carbenoxolone (CBX), to the LGN via the proximal microdialysis probe. As predicted by studies in acute LGN slices (Hughes et al., 2004; Lorincz et al., 2008), CBX significantly and reversibly reduced the synchrony of $\alpha$ rhythm-related firing in both HT burst-HT burst cell pairs (cross-correlogram peak, \% of control: $28.1 \pm 8.4 \%$; $p<0.01 ; n=3$ ) and HT burst-tonic cell pairs (cross-correlogram peak, \% of control: $36.29 \pm 6.8 \% ; p<0.05 ; n=4$; Figures 3A,B).

\section{DIRECT ULTRASTRUCTURAL EVIDENCE FOR GJS BETWEEN THALAMOCORTICAL NEURONS}

Although the in vivo data presented here, along with previous work carried out in vitro, strongly suggests that TC neurons are interconnected by GJs, unequivocal anatomical evidence for these structures has thus far been lacking. To address this, we examined ultrathin sections from the LGN of adult cats using both conventional and freeze-fracture EM. Observations of these sections revealed an abundance of GJs with characteristic heptalaminar structure throughout the LGN. Using well established ultrastructural criteria (Peters et al., 1991), we identified such junctions involving both glial and neuronal processes. GJs were most frequently observed between astrocytic processes, followed by junctions between astrocytic processes and neuronal dendrites. Astrocytic processes, which contained few or no organelles, were characteristically irregular and closely molded to adjacent neuropil elements. Of the GJs that exclusively occurred between neuronal elements, those between dendrites were the most common (Figures $4 \mathbf{A 1}, \mathbf{A} 2$ ). The prevalence of GJs in the LGN was confirmed in freeze-fracture preparations. Examination of replicas in these preparations revealed junctional plaques of varying sizes with different arrangements of intramembranous particles. These preparations as well as thin-section EM showed the consistent presence of GJs between dendrites and synapse-forming axon terminals (Figures 4B1,B2).

\section{PHENOTYPIC EVIDENCE OF GJ COUPLING IS COMMONLY OBSERVED IN RECORDINGS OF TC NEURONS IN VITROBUT NEVER IN RECORDINGS OF LOCAL CIRCUIT INTERNEURONS}

Although we were unable to establish with absolute certainty using EM whether the dendrites involved in the formation of GJs in the LGN belonged to TC neurons or interneurons, the absence of presynaptic specializations or clustered vesicles, features that characterize the latter neuronal cell type, suggests that they were specific to TC neurons. To add further confidence to this suggestion, we assessed the relative prevalence of phenotypic characteristics 


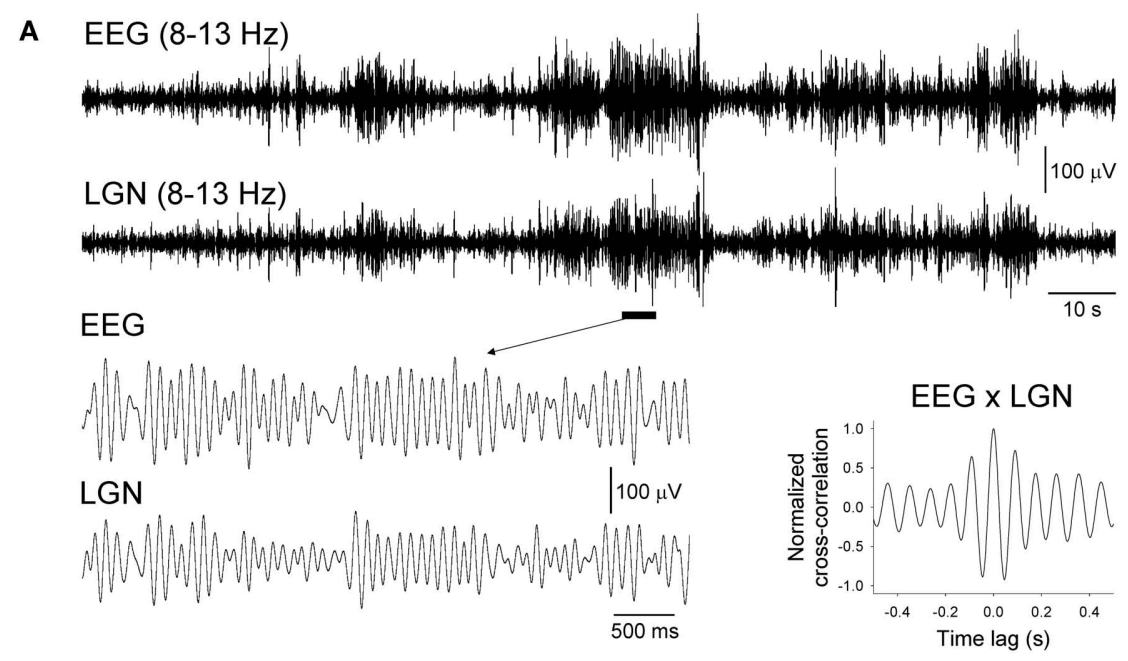

B 1

1 HT bursting TC neuron
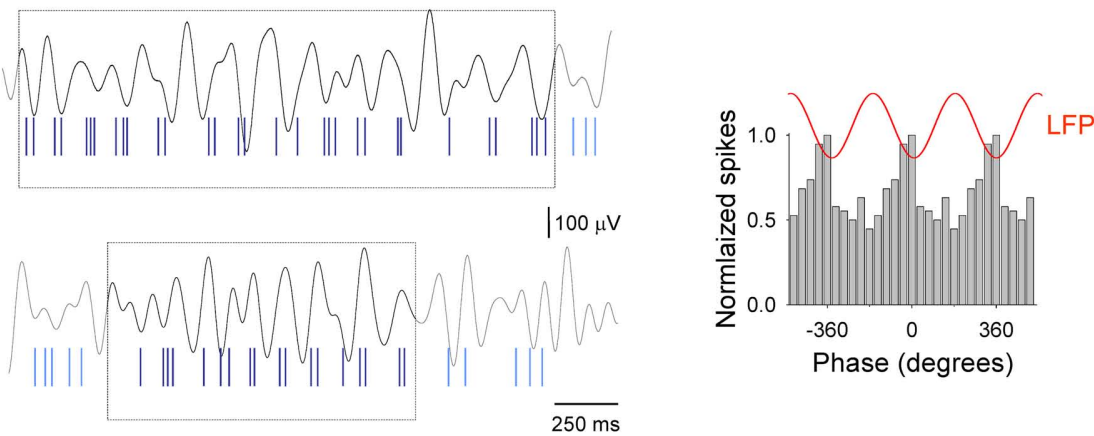

2

Relay-mode TC neuron

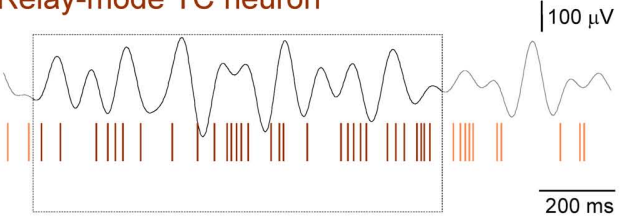

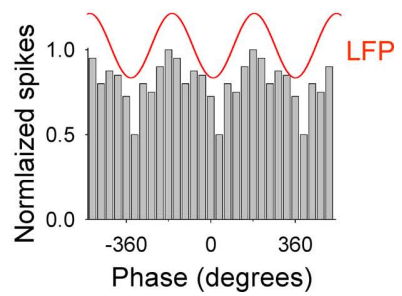

FIGURE 1 | Basic appearance of $\alpha$ rhythms in the EEG and LGN and associated firing patterns of LGNTC neurons. (A) Simultaneous recordings of the occipital EEG (top) and LFP in the LGN (bottom) during a period of quiet wakefulness in a freely moving cat. Both signals are filtered at $8-13 \mathrm{~Hz}$ to reveal a pattern of waxing and waning $\alpha$ rhythms. The underlined section is enlarged below and shows more clearly an individual epoch of $\alpha$ activity. Synchrony between the EEG and LGN is illustrated by the corresponding cross-correlogram which is shown to the bottom right (computed from the traces in the top panel). (B1) Activity of a presumed HT bursting TC neuron in the LGN during two brief episodes of $\alpha$ activity (marked by the boxes in the top and bottom panels). The top trace in each panel is the LGN LFP. Note how brief bursts of two or three spikes predominantly occur close to the negative peaks of the LFP (top panel) although they can also occasionally switch to occurring near the positive peaks (lower panel). The corresponding spike-timing histogram for several consecutive $\alpha$ rhythm epochs is shown to the right. The red trace above this plot is a schematic representative of the oscillatory signal in the LFP. (B2) Activity of a relay-mode LGNTC neuron during a brief $\alpha$ rhythm epoch (identified by the box). Again, the top trace is the LGN LFP. The corresponding spike-timing histogram for several consecutive $\alpha$ rhythm epochs is shown to the right and reveals that firing in this neuron is suppressed close to the negative LFP peak (for additional details relating to the activity of LGNTC neurons during $\alpha$ rhythms see Lorincz et al., 2009b). suggestive of GJ expression for both TC neurons and interneurons in a parallel set of experiments carried out using intracellular recording in cat LGN slices. In particular, we examined, (i) the proportion of each cell type associated with dye-coupling, and (ii) the percentage of each cell type exhibiting spikelets (i.e., action potentials presumed to be electrotonically communicated via GJs).
Overall, 31\% (30 of 96) of LGN TC neurons are associated with dye-coupling (Figures 5A,C). This dye-coupling most often involves either two or three cells (e.g., Figure 5A; see Hughes et al., 2002a) and starts to appear prominently at around 5-6 weeks (Figure 5C). Importantly, dye-coupling between TC neurons is fully prevented when intracellular recording and dye injection were performed in 

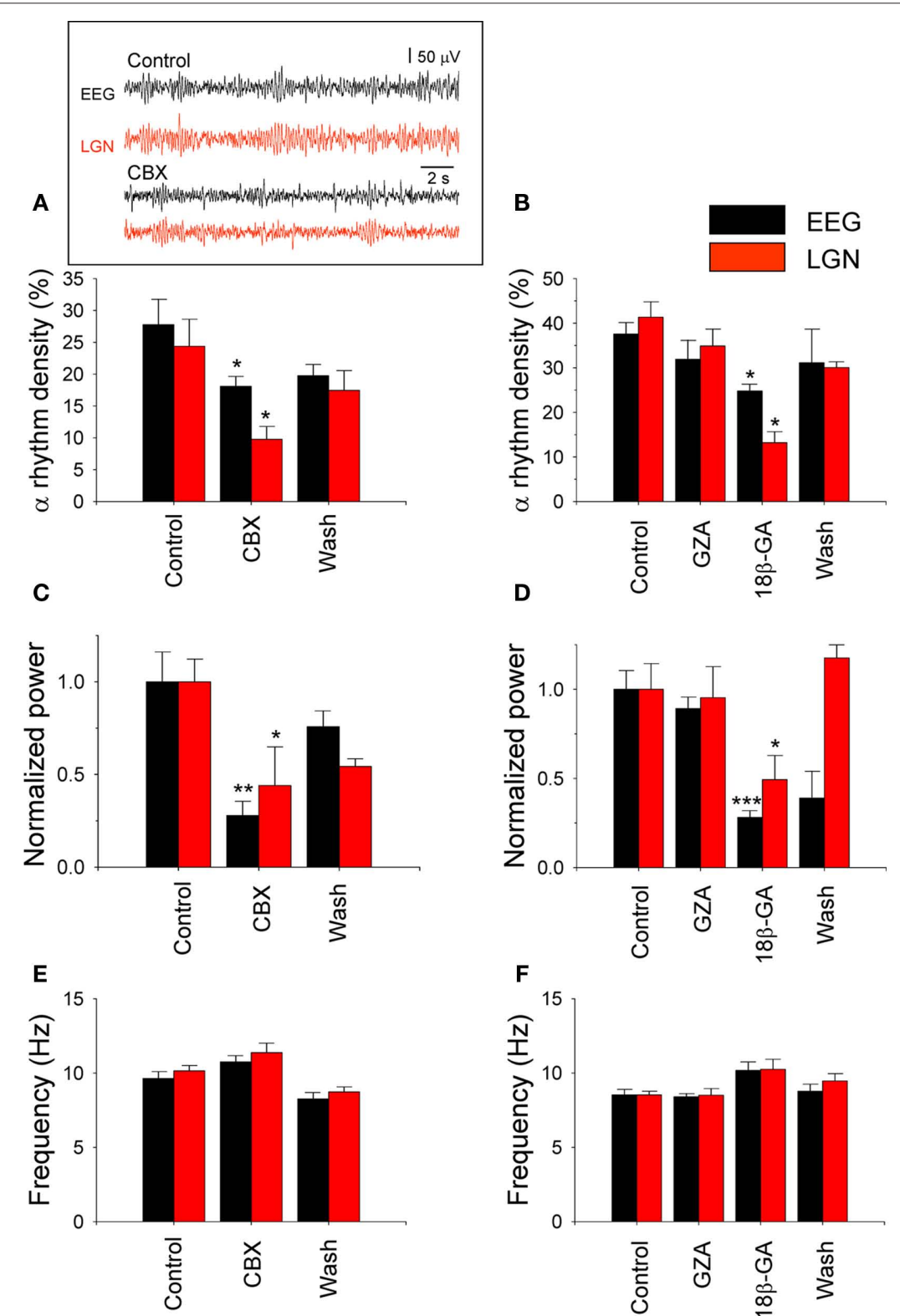

FIGURE 2 |Thalamic delivery of gap junction (GJ) blockers disrupts LGN and EEG a rhythms. (A-F) Bar graphs summarizing the effect of thalamic CBX and GZA/18ß-GA application on EEG (black bars) and LGN (red bars) $\alpha$ rhythm density (A,B, respectively), normalized power (C,D, respectively), and frequency
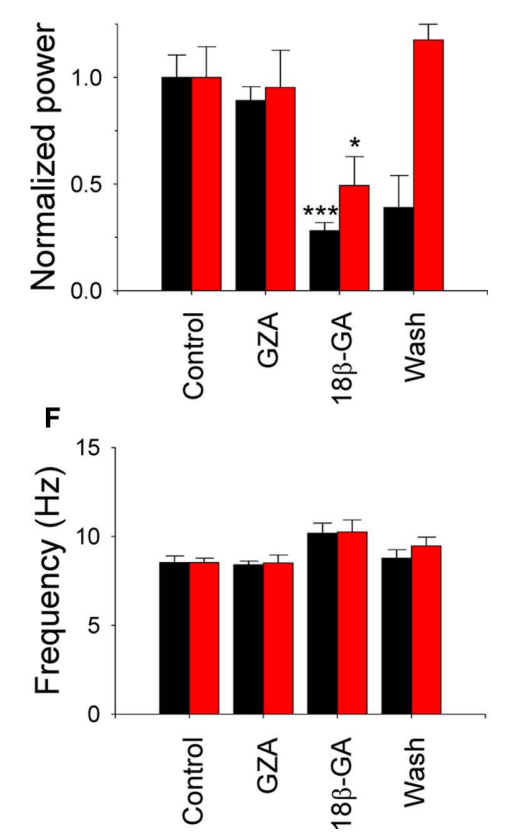

(E,F, respectively; see also Table 1; note: ACSF was delivered to the LGN during the Control andWash periods; ${ }^{*} p<0.05 ;{ }^{* *} p<0.01$; $\left.{ }^{* *} p<0.001\right)$. The inset in panel (A) shows representative EEG (black traces) and LGN LFP (red traces) recordings during a control period (top) and following CBX administration (bottom).

the continuous presence of $100 \mu \mathrm{M} \mathrm{CBX}(n=0 / 18)$. Consistent with our EM results dye-coupling between TC neurons was rarely observed at soma-to-soma sites (two cases) and usually involved at least one dendritic process (mean distance between somas of coupled neurons: $62.2 \pm 5.8 \mu \mathrm{m} ; n=27$ pairs). In stark contrast to TC neurons, interneurons were not associated with dye-coupling in any cases ( $n=0$ of 14 ; e.g., Figure $5 B)$.

In total, $19 \%$ (39 of 205) of LGN TC neurons exhibit spikelets (time-to-peak: $1.2 \pm 0.1 \mathrm{~ms}$; amplitude; $2.1 \pm 0.2 \mathrm{mV}$; t decay: $3.9 \pm 0.5 \mathrm{~ms} ; n=60$ events from 20 neurons; Figures 6A1,C). These spikelets are resistant to the application of blockers of fast glutamatergic transmission $(20 \mu \mathrm{M}$ NBQX to block AMPA/kainate receptors and $100 \mu \mathrm{M}$ APV to block NMDA receptors, $n=4$; not illustrated) but are abolished by $100 \mu \mathrm{M} \mathrm{CBX}$ ( $n=4$; Figure 6A1; see also Hughes et al., 2002a). CBX had no effect on the resting membrane potential or the apparent input resistance of LGN TC neurons ( $p>0.5$; $n=3$; Figure 6A2), ruling out the possibility that non-specific actions on other membrane properties by CBX could explain the block of spikelets. Interneurons did not exhibit spikelets in any cases $(n=0$ of 30$)$. These neurons did exhibit spontaneous subthreshold potentials but these were blocked in all cases by a combination of NBQX and APV and possessed a waveform that clearly 
Table 1 | Effect of thalamic CBX, 18 $\beta$-GA, and GZA application on $\alpha$ rhythm density and power in vivo.

\begin{tabular}{|c|c|c|c|c|}
\hline & \multicolumn{2}{|c|}{ LGN } & \multicolumn{2}{|c|}{ EEG } \\
\hline & Density (\%) & $\begin{array}{l}\text { Power } \\
\left(\times 1000 \mu V^{2}\right)\end{array}$ & Density (\%) & $\begin{array}{l}\text { Power } \\
\left(\times 1000 \mu V^{2}\right)\end{array}$ \\
\hline Control $(n=30)$ & $24.38 \pm 4.21$ & $1.93 \pm 0.23$ & $27.76 \pm 4.03$ & $3.80 \pm 0.61$ \\
\hline 1 mM CBX & $9.78 \pm 1.94 *$ & $0.85 \pm 0.40^{*}$ & $18.11 \pm 1.49^{*}$ & $1.26 \pm 0.29 * *$ \\
\hline Wash & $17.45 \pm 3.12$ & $1.048 \pm 0.08$ & $19.75 \pm 1.71$ & $2.88 \pm 0.32$ \\
\hline Control $(n=30)$ & $41.35 \pm 3.47$ & $2.33 \pm 0.33$ & $37.56 \pm 2.51$ & $6.89 \pm 0.72$ \\
\hline $1 \mathrm{mM}$ GZA & $34.89 \pm 3.82$ & $2.22 \pm 0.40$ & $31.9 \pm 4.33$ & $6.15 \pm 0.44$ \\
\hline $1 \mathrm{mM} 18 \beta-\mathrm{GA}$ & $13.21 \pm 2.46^{*}$ & $1.15 \pm 0.31 *$ & $24.8 \pm 1.42^{*}$ & $1.94 \pm 0.26 * * *$ \\
\hline Wash & $30.09 \pm 1.26$ & $2.74 \pm 0.72$ & $31.14 \pm 7.46$ & $2.69 \pm 1.04^{* *}$ \\
\hline
\end{tabular}

$\left({ }^{*} p<0.05 ;{ }^{*} p<0.01 ;{ }^{* *} p<0.001\right)$. identified them as conventional EPSPs (time-to-peak: $4.4 \pm 0.2 \mathrm{~ms}$; amplitude; $4.6 \pm 0.4 \mathrm{mV} ; \tau$ decay: $32.4 \pm 2.5 \mathrm{~ms} ; n=100$ events from 10 neurons; Figures 6B,C; Cox et al., 2003; Lorincz et al., 2009b). Thus, whilst TC neurons commonly exhibit phenotypic features consistent with GJ expression, interneurons never do.

\section{DISCUSSION}

The main findings of this study are: (i) direct delivery of either of the putative GJ blockers, CBX or 18 $\beta$-GA, to the LGN of freely moving cats using reverse microdialysis reduces both the density and power of LGN and EEG $\alpha$ rhythms, whereas the glycyrrhetinic acid derivative that is inactive as GJ blocker, GZA, is without effect, (ii) local delivery of CBX to the LGN suppresses $\alpha$ rhythmrelated synchronized firing in both HT bursting TC neuron pairs and HT bursting-tonic/relay-mode firing pairs, (iii) examination of ultrathin sections of the LGN from adult cats using both

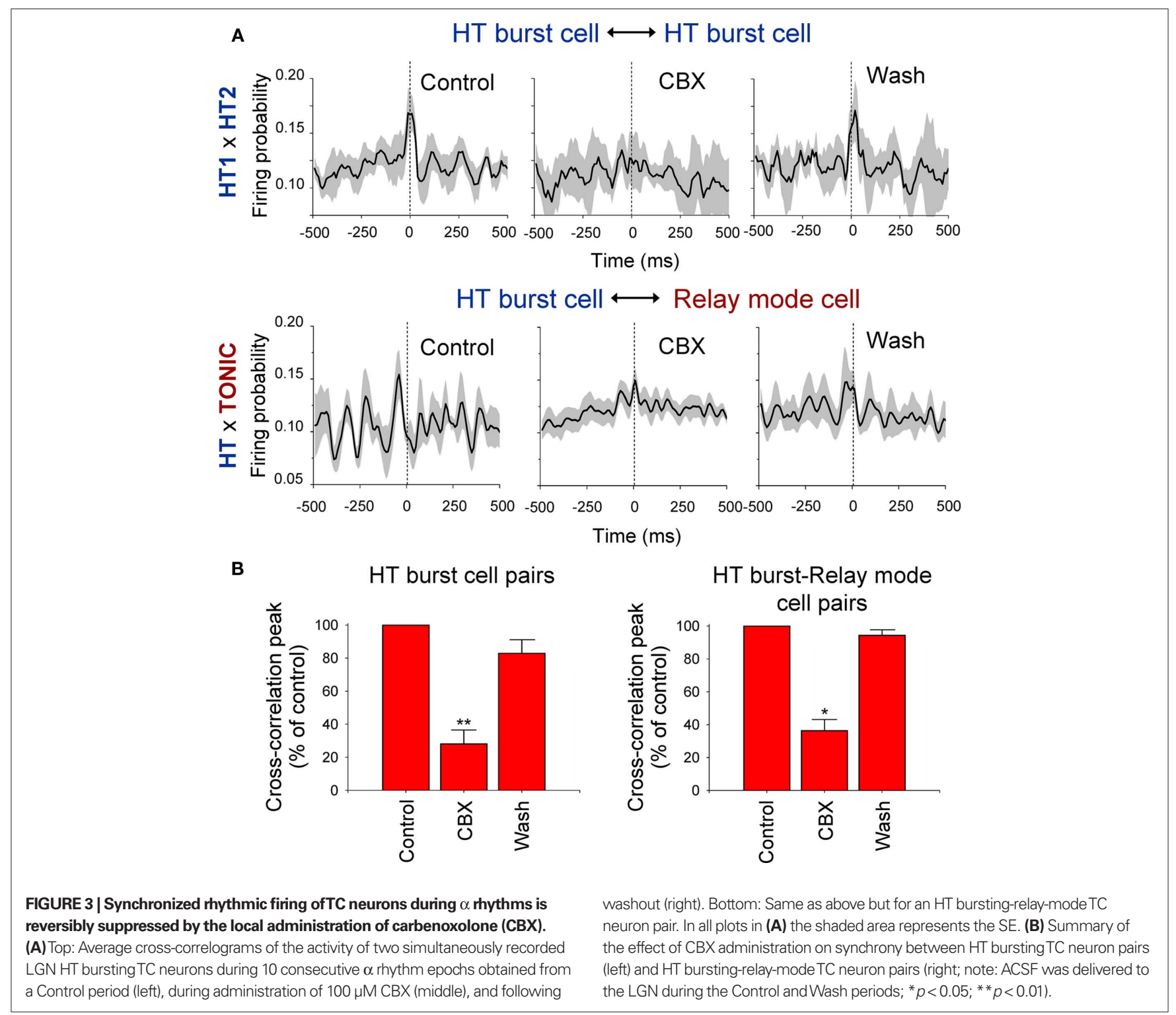




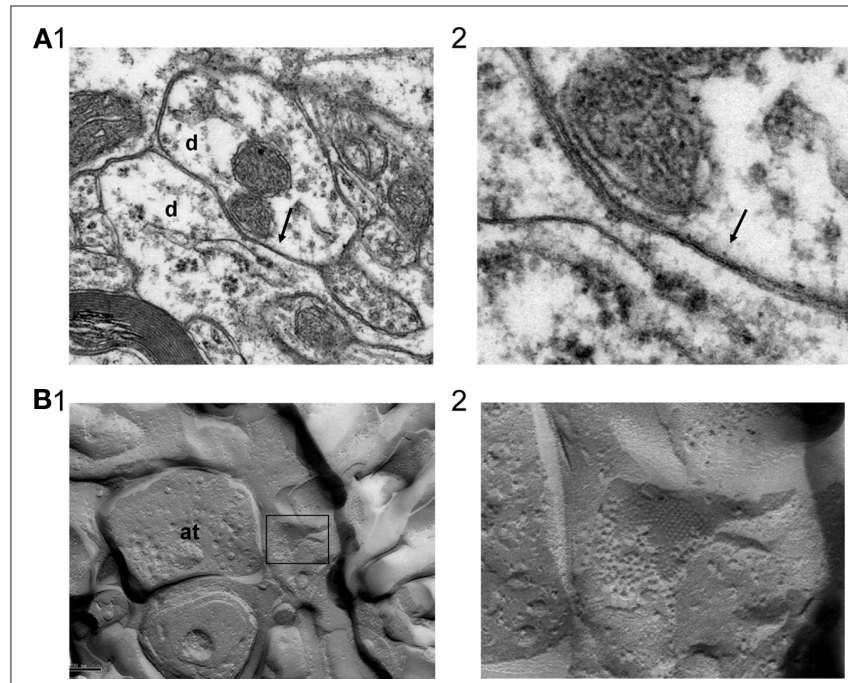

FIGURE 4 | Ultrastructural evidence for GJs between neurons in the cat LGN. (A) GJ (arrow) formed between two dendritic elements (labeled " $d$ ") in the cat LGN (A1). The junction is shown at higher magnification in (A2). (B) Example of a gap junctional plaque, contained within the box, in close association with a synapse-forming axon terminal (labeled "at") (B1). The plaque is shown at higher magnification in (B2).

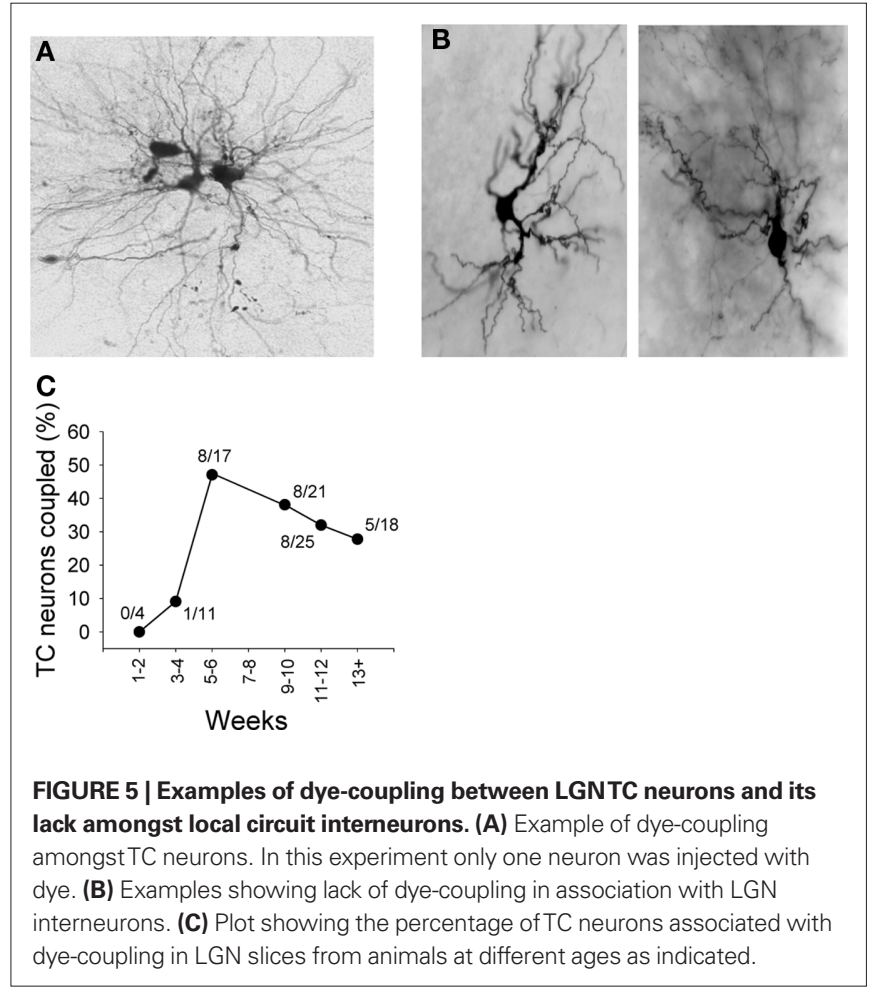

conventional and freeze-fracture EM reveals that GJs between neuronal processes are commonplace, (iv) assessment of the relative proportions of the phenotypic GJ markers, dye-coupling, and spikelets, in TC neurons and interneurons reveals that whilst they are frequently encountered with respect to TC neurons they are completely absent in relation to interneurons, indicating that the GJs detected using EM almost certainly belong to TC neurons.

\section{A ROLE FOR GJS IN CONTROLLING $\alpha$ RHYTHMS IN THE INTACT BRAIN}

Although an abundance of studies in acute brain slice preparations have indicated a role for GJs in shaping the properties of physiologically relevant network oscillations (Connors and Long, 2004), in vivo evidence supporting this concept is extremely limited and largely restricted to isolated observations of spikelets in intracellular recordings obtained from anesthetized animals (Deschenes et al., 1984; Steriade et al., 1991; Timofeev and Steriade, 1997; see also Epsztein et al., 2010) and a small number of studies in connexin-36 (Cx36) knock-out (KO) mice (e.g., Hormuzdi et al., 2001). Since neither mice, nor indeed rats, exhibit genuine equivalents to the robust occipital $\alpha$ activity that is present in larger mammals, using $\mathrm{KO}$ animals is not a viable approach for dissecting the mechanisms of such activity. Thus the current endeavor is one of the few to address the role of GJs in controlling physiological brain oscillations in the whole animal and to our knowledge the only study to do so in non-rodents.

One of the obvious drawbacks to using so-called GJ blockers such as CBX and 18 $\beta$-GA is their well-documented lack of selective action at GJs (e.g., Rouach et al., 2003). Whilst we fully acknowledge this issue, two main arguments support the view that the in vivo effects observed with these agents in this study can be ascribed to a genuine inhibition of GJ-mediated signaling. First, the glycyrrhetinic derivative, GZA, which shares much of its pharmacology with CBX and 18ß-GA but which lacks effects on GJs is ineffective on LGN and EEG $\alpha$ rhythms. Second, the effects of CBX in thalamic slices described here in preventing dye-coupling and blocking spikelets are fully consistent with an inhibition of GJ signaling. Furthermore, the lack of discernible effects of CBX on other membrane properties suggests that its thalamic effects are confined to an action on GJs. Another potential confound in this study is the possibility of passive spread of GJ blockers to other areas such as the nucleus reticularis thalami (NRT) and neocortex. Whilst we cannot fully discount that such spread may have influenced our results we believe it is unlikely, given the correspondence between in vivo and in vitro data, that the observations in the LGN would be due to an indirect effect of GJ blockers in other areas.

\section{DIRECT EVIDENCE OF GJS IN THE CAT LGN AND CORRESPONDENCE WITH PREVIOUS STUDIES}

Our results show unequivocally for the first time that GJ structures are present between neurons in the cat LGN. Given that, (i) there is an absence of presynaptic specializations or clustered vesicles in the neuronal processes with which these GJs are associated, and (ii) there is an abundance of phenotypic evidence for GJ coupling between TC neurons but none whatsoever for such coupling between LGN interneurons, we can only reasonably conclude that these GJs belong to TC neurons. This assertion is fully consistent with several of our previous studies which detail extensive evidence for GJ coupling between LGN TC neurons (Hughes et al., 2002a, 2004; Hughes and Crunelli, 2007; Blethyn et al., 2008; Lorincz et al., 2008, 2009a,b).

Recently, by recording from pairs of TC neurons in the rat and mouse ventrobasal (VB) thalamus Lee et al. (2010) also directly demonstrated the presence of GJ-based electrical synapses between TC neurons. Unlike in the cat LGN, electrophysiological evidence 
A 1

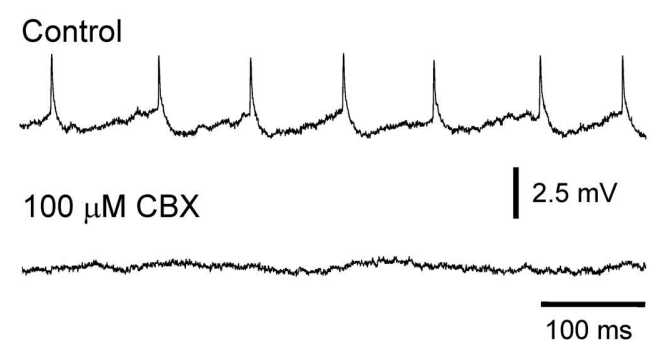

2

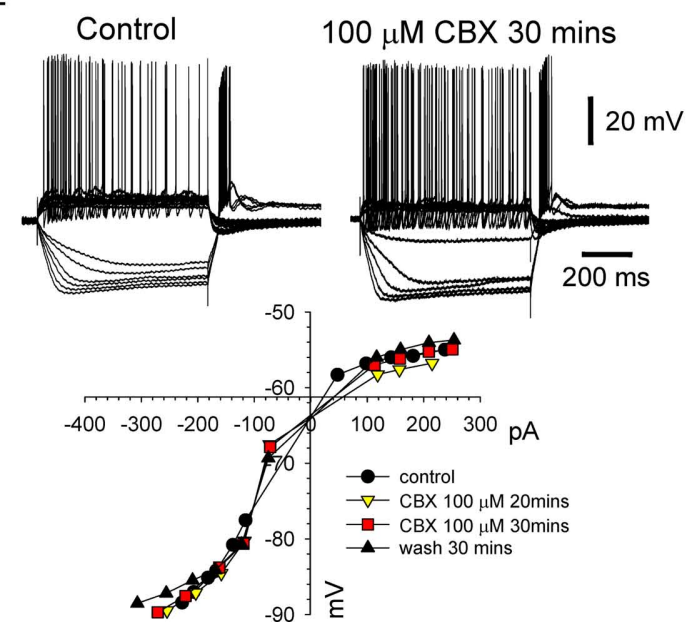

B

\section{Interneuron}

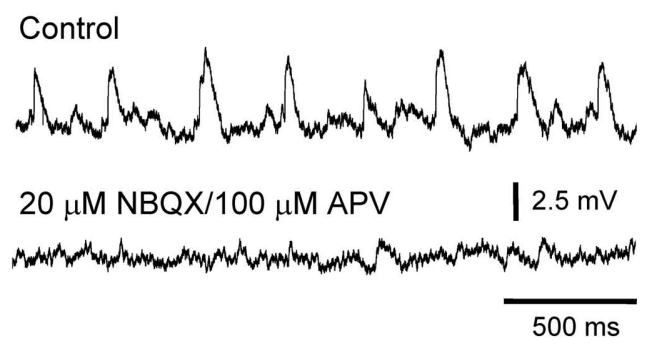

C

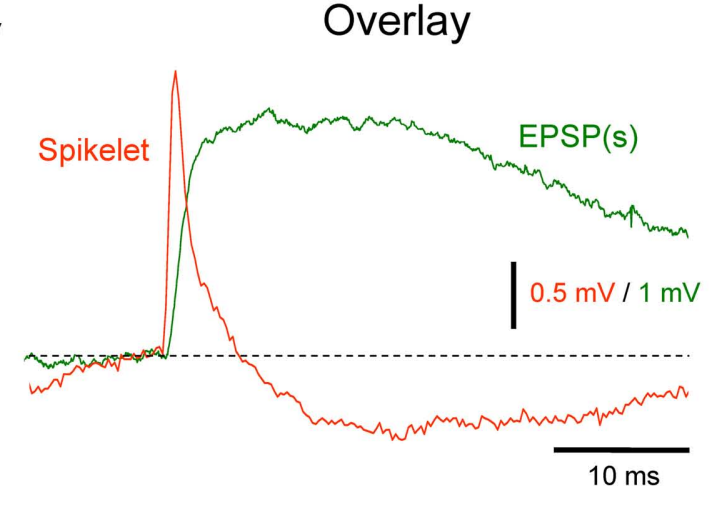

properties. (B) Intracellular recording of an LGN interneuron showing spontaneous rhythmic potentials (top) which are blocked by a combination of $20 \mu \mathrm{M}$ NBQX and $100 \mu \mathrm{M}$ APV (bottom) confirming that they are conventional, glutamatergic EPSPs. (C) Overlay of an average TC neuron spikelet (red trace) and interneuron EPSP (green trace). Each trace is the average of 10 individual events from the recordings shown in $(\mathbf{A 1}, \mathbf{B})$. Note the clearly distinct waveforms of the two events. for GJs in the rodent VB is most prominent in the first postnatal week and then gradually disappears by the end of the second week, a sequence which is paralleled by a reduction in Cx36 expression. Although this would appear to imply that GJs in the rodent VB are only present during development the authors are careful to point out, (i) that another connexin protein or proteins is also involved since some electrical synapses are also present in developing $\mathrm{Cx} 36 \mathrm{KO}$ mice, and, (ii) that the large decrease in apparent input resistance that occurs at the end of the second postnatal week may occlude the detection of GJs with weak junctional conductance. This latter point is especially interesting because in TC neurons recorded intracellularly from the cat LGN and VB, electrophysiological evidence of GJs (in this case spikelets) is rarely observed without bringing about a substantial increase in apparent input resistance through the pharmacological activation of either Group I metabotropic glutamate receptors (mGluRs; Hughes et al., 2002a, 2004; Lorincz et al., 2009a) or muscarinic Ach receptors (mAchRs; Lorincz et al., 2008, 2009a,b). Thus, it may be that in adult rats and mice, a similar activation of mGluRs and mAchRs is required to observe the electrophysiological effects of GJs. Such a suggestion is consistent with ultrastructural studies showing that neurons in the adult rat VB exhibit membrane appositions which resemble GJs (Lieberman and Spacek, 1997). All this said, and regardless of whether or not TC neurons from the VB of adult rats and mice express GJs, a degree of caution should be exercised when attempting to relate findings from rodents to those obtained from cats.

\section{PHYSIOLOGICAL RELEVANCE}

The involvement of GJs in $\alpha$ rhythms has significant consequences for interpreting the macroscopic changes in these oscillations that are known to correlate with many aspects of perception, cognition, and memory (see Introduction). One key aspect that we have highlighted in recent work is the ability of GJs to facilitate both in-phase and anti-phase synchronization of TC neuron firing. As such, at any given time, the amplitude of $\alpha$ rhythms reflects the net effect of groups of HT bursting neurons whose phase relationships are dynamically changing and may at times be in direct competition (i.e., in the case of an anti-phase relationship; Hughes and Crunelli, 2007). An important upshot of this is that a reduction in macroscopic $\alpha$ rhythm amplitude, as typically occurs for the 
dominant occipital $\alpha$ rhythm upon eye opening, may not simply reflect a reduction in the basic oscillatory activity of underlying pacemaker neurons (i.e., HT bursting cells) but a reconfiguring of phase relationships between these cells such that the overall output is characterized by destructive interference (Hughes and Crunelli, 2007). It is easy to foresee that such a dramatic phase reorganization could readily occur in response to the perturbation of the HT bursting cell network that would result from a sudden burst of excitatory input from the retina (e.g., see Vervaeke et al., 2010). It also not difficult to envisage how more subtle changes in the basic level of excitability in the LGN can cause changes in ongoing phase relationships between GJ-coupled constituent neurons and ultimately be reflected at a more macroscopic level (Hughes and Crunelli, 2007).

Whilst evidence for such dynamic phase shifting is clear from experiments in cat LGN slices (Hughes et al., 2004; Hughes and Crunelli, 2007; Lorincz et al., 2008, 2009b), and to some degree apparent in in vivo experiments in this species (see Figure $\mathbf{1}$ in Lorincz et al., 2009b), there is also evidence from depth recordings in humans that macroscopic EEG $\alpha$ rhythms may reflect the global summation of more fragmented, localized rhythms whose phase relationships between each other are dynamically changing and, more specifically, shifting between in-phase and anti-phase synchronicity (Cohn, 1948). Of course, the idea that

\section{REFERENCES}

Adrian, E. D., and Matthews, B. H. C. (1934). The Berger rhythm: potential changes from the occipital lobes of man. Brain 57, 355-385.

Bartley, S. H. (1940). The relationship between cortical responses to visual stimulation and changes in the alpha rhythm. J. Exp. Psychol. 27, 624-639.

Berger, H. (1929). Über das Elektroenkephalogramm des Menchen. Arch. Psychiatr. Nervenkr. 87, 527-570.

Berman, A. L., and Jones, E. G. (1982). The Thalamus and Basal Telencephalon of the Cat. Madison, WI: The University of Wisconsin Press.

Blethyn, K. L., Hughes, S. W., and Crunelli, V. (2008). Evidence for electrical synapses between neurons of the nucleus reticularis thalami in the adult brain in vitro. Thalamus Relat. Syst. 4, 13-20.

Busch, N. A., Dubois, J., and VanRullen, R. (2009). The phase of ongoing EEG oscillations predicts visual perception. J. Neurosci. 29, 7869-7876.

Chatila, M., Milleret, C., Buser, P., and Rougeul, A. (1992). A $10 \mathrm{~Hz}$ "alphalike" rhythm in the visual cortex of the waking cat. Electroencephalogr. Clin. Neurophysiol. 83, 217-222.

Chatila, M., Milleret, C., Rougeul, A., and Buser, P. (1993). Alpha rhythm in the cat thalamus. C. R. Acad. Sci. III 316, 51-58.

Cohn, R. (1948). The occipital alpha rhythm: a study of phase variations. J. Neurophysiol. 11, 31-37.
Connors, B. W., and Long, M. A. (2004). Electrical synapses in the mammalian brain. Annu. Rev. Neurosci. 27, 393-418.

Cox, C. L., Reichova, I., and Sherman, S. M. (2003). Functional synaptic contacts by intranuclear axon collaterals of thalamic relay neurons. J. Neurosci. 23, 7642-7646.

Crick, F., and Koch, C. (2003). A framework for consciousness. Nat. Neurosci. 6, 119-126.

Crunelli, V., Leresche, N., and Parnavelas, J. G. (1987). Membrane properties of morphologically identified $\mathrm{X}$ and $\mathrm{Y}$ cells in the lateral geniculate nucleus of the cat in vitro. J. Physiol. (Lond.) 390, 243-256.

Davidson, J. S., and Baumgarten, M. (1988). Glycyrrhetinic acid derivatives: a novel class of inhibitors of gap-junctional intercellular communication: structure activity relationships. J. Pharmacol.Exp. Ther. 246, 1104-1107.

Deschenes, M., Paradis, M., Roy, J. P., and Steriade, M. (1984). Electrophysiology of neurons of lateral thalamic nuclei in cat: resting properties and burst discharges. J. Neurophysiol. 51, 1196-1219.

Domich, L., Oakson, G., and Steriade, M. (1986). Thalamic burst patterns in the naturally sleeping cat: a comparison between cortically projecting and reticularis neurones. J. Physiol. (Lond.) 379, 429-449.

Efron,E.(1970). The minimum duration of a perception. Neuropsychologia 8,57-63.

Epsztein, J., Lee, A. K., Chorev, E., and Brecht, M. (2010). Impact of spikelets

changes in EEG $\alpha$ rhythm amplitudes reflect a re-organization of phase relationships between interconnected underlying oscillators is an old one and was essentially first proposed by Adrian and Matthews (1934) over 75 years ago in their seminal publication which confirmed and extended Berger's findings. In this paper they suggest that the occipital $\alpha$ rhythm is due to the spontaneous rhythmic activity of cells that "beat synchronously" when undisturbed but which can be "broken up" by exposing the cells to a "mosaic of excitations" of either a visual or nonvisual nature. However, the GJ-coupled network of intrinsically rhythmic thalamic neurons (i.e., HT bursting cells) described here and in our previous studies (Hughes et al., 2004; Hughes and Crunelli, 2007; Lorincz et al., 2008, 2009b) provides a firm basis for this idea. Lastly, given that according to the scheme outlined in the introduction, any changes in $\alpha$ rhythm generation at the thalamic level will necessarily affect the basic temporal properties of relay-mode throughput, it is a small step to begin to imagine how they may ultimately impinge upon the temporal aspects of early stage perceptual processing.

\section{ACKNOWLEDGMENTS}

This work was supported by the Wellcome Trust grants 71436, 78403, 91882 awarded to Vincenzo Crunelli and 78311 awarded to Stuart W. Hughes.

on hippocampal CA1 pyramidal cell activity dueing spatial exploration. Science 327, 474-477.

Fingelkurts, A. A., and Fingelkurts, A. A (2006). Timing in cognition and EEG brain dynamics: discreteness versus continuity. Cogn. Process. 7, 135-162.

Hormuzdi, S. G., Pais, I., LeBeau, F. E., Towers, S. K., Rozov, A., Buhl, E. H., Whittington, M. A., and Monyer, $\mathrm{H}$. (2001). Impaired electrical signalling disrupts gamma frequency oscillations in connexin 36-deficient mice. Neuron 31, 487-495.

Hughes, S. W., Blethyn, K. L., Cope, D. W. and Crunelli, V. (2002a). Properties and origin of spikelets in thalamocortical neurones in vitro. Neuroscience 110, 395-401.

Hughes, S. W., Cope, D. W., Blethyn, K. L., and Crunelli, V. (2002b). Cellular mechanisms of the slow $(<1 \mathrm{~Hz})$ oscillation in thalamocortical neurons in vitro. Neuron 33, 947-958.

Hughes, S. W., and Crunelli, V. (2005). Thalamic mechanisms of EEG alpha rhythms and their pathological implications. Neuroscientist 11, 357-372.

Hughes, S. W., and Crunelli, V. (2007) Just a phase they're going through: the complex interaction of intrinsic high-threshold bursting and gap junctions in the generation of thalamic alpha and theta rhythms. Int. J. Psychophysiol. 64, 3-17.

Hughes, S. W., Lorincz, M., Cope, D. W. Blethyn, K. L., Kekesi, K. A., Parri, H. R., Juhasz, G., and Crunelli, V. (2004).
Synchronized oscillations at alpha and theta frequencies in the lateral geniculate nucleus. Neuron 42, 253-268.

James, W. (1890). Principles of Psychology, Vol. 1. New York, NY: Holt.

Kristofferson,A.B. (1967). Successiveness discrimination as a two-state, quantal process. Science 158, 1337-1339.

Lansing, R. W. (1957). Relation of brain and tremor rhythms to visual reaction time. Electroencephalogr. Clin. Neurophysiol. 9, 487-504.

Lee, S. C., Cruikshank, S. J., and Connors, B. W. (2010). Electrical and chemical synapses between relay neurons in developing thalamus. J. Physiol. (Lond.) 588, 2403-2415.

Lieberman, A. R., and Spacek, J. (1997). Filamentous contacts: the ultrastructure and three-dimensional organization of specialized non-synaptic interneuronal appositions in thalamic relay nuclei. Cell Tissue Res. 288, 43-57.

Lindsley, D. B. (1952). Psychological phenomena and the electroencephalogram. Electroencephalogr. Clin. Neurophysiol. 4, 443-456.

Lopes da Silva, F. H., van Lierop, T. H., Schrijer, C. F., and van Leeuwen, W. S. (1973a). Essential differences between alpha rhythms and barbiturate spindles: spectra and thalamo-cortical coherences. Electroencephalogr. Clin. Neurophysiol. 35, 641-645.

Lopes da Silva, F. H., van Lierop, T. H., Schrijer, C. F., and van Leeuwen, W. S. (1973b). Organization of thalamic and cortical alpha rhythms: spectra 
and coherences. Electroencephalogr. Clin. Neurophysiol. 35, 627-639.

Lorincz, M. L., Crunelli, V., and Hughes, S. W. (2008). Cellular dynamics of cholinergically induced alpha $(8-13 \mathrm{~Hz})$ rhythms in sensory thalamic nuclei in vitro. J. Neurosci. 28, 660-671.

Lorincz, M. L., Geall, F., Bao, Y., Crunelli, V., and Hughes, S. W. (2009a). ATPdependent infra-slow $(<0.1 \mathrm{~Hz})$ oscillations in thalamic networks. PLoS ONE 4, e4447. doi: 10.1371/journal. pone.0004447

Lorincz, M. L., Kekesi, K. A., Juhasz, G., Crunelli, V., and Hughes, S. W. (2009b). Temporal framing of thalamic relay-mode firing by phasic inhibition during the alpha rhythm. Neuron 63, 683-696.

Mathewson, K. E., Gratton, G., Fabiani, M., Beck, D. M., and Ro, T. (2009). To see or not to see: prestimulus alpha phase predicts visual awareness. $J$. Neurosci. 29, 2725-2732.

McCormick, D. A., and Pape, H.C. (1988). Acetylcholine inhibits identified interneurons in the cat lateral geniculate nucleus. Nature 334, 246-248.

Nunn, C. M. H., and Osselton, J. W. (1974). The influence of the EEG alpha rhythm on the perception of visual stimuli. Psychophysiology 11, 294-303.
Pape, H.-C., and McCormick, D. A. (1995). Electrophysiological and pharmacological properties of interneurons in the cat dorsal lateral geniculate nucleus. Neuroscience 68, 1105-1125.

Peters, A., Palay, S. L., and Webster, H. F. (1991). Fine Structure of the Nervous System. Oxford: Oxford University Press.

Pirchio, M., Turner, J. P., Williams, S. R., Asprodini, E., and Crunelli, V. (1997). Postnatal development of membrane properties and delta oscillations in thalamocortical neurons of the cat dorsal lateral geniculate nucleus. $J$. Neurosci. 17, 5428-5444.

Rouach, N., Segal, M., Koulakoff, A., Giaume, C., and Avignone, E. (2003). Carbenoxolone blockade of neuronal network activity in culture is not mediated by an action on gap junctions. J. Physiol. (Lond.) 15, 729-745.

Rougeul-Buser, A., and Buser, P. (1997). Rhythms in the alpha band in cats and their behavioural correlates. Int. J. Psychophysiol. 26, 191-203.

Steriade, M., Dossi, R. C., Pare, D., and Oakson, G. (1991). Fast oscillations $(20-40 \mathrm{~Hz})$ in thalamocortical systems and their potentiation by mesopontine cholinergic nuclei in the cat. Proc. Natl. Acad. Sci. U.S.A. 88, 4396-4400.
Stroud, J. M. (1955). “The fine structure of psychological time," in Information Theory in Psychology, ed. H. Quastler (Glencoe, IL: Free Press), 140-207.

Surwillo, W. W. (1961). Frequency of the "alpha" rhythm, reaction time and age. Nature 191, 823-824.

Timofeev, I., and Steriade, M. (1997). Fast (mainly 30-100 Hz) oscillations in the cat cerebellothalamic pathway and their synchronization with cortical potentials. J. Physiol. (Lond.) 504, 153-168.

VanRullen, R., and Koch, C. (2003). Is perception discrete or continuous? Trends Cogn. Sci. (Regul. Ed.) 7, 207-213.

Varela, F. J., Toro, A., John, E. R., and Schwartz, E. L. (1981). Perceptual framing and cortical alpha rhythm. Neuropsychologia 19, 678-686.

Vervaeke, K., Lorincz, A., Gleeson, P., Farinella, M., Nusser, Z., and Silver, R.A. (2010). Rapid desynchronization of an electrically-coupled interneuron network with sparse excitatory input. Neuron 67, 435-451.

Wiener, N. (1985). "Time and organization," in Norbert Wiener: collected works, Vol.IV, ed.P.Masani (Cambridge, MA: MIT Press).

Williams, S. R., Turner, J. P., Anderson, C. M., and Crunelli, V. (1996) Electrophysiological and morphological properties of interneurones in the rat dorsal lateral geniculate nucleus in vitro. J. Physiol. (Lond.) 490, 129-147.

Conflict of Interest Statement: The authors declare that the research was conducted in the absence of any commercial or financial relationships that could be construed as a potential conflict of interest.

Received: 15 March 2011; paper pending published: 25 April 2011; accepted: 27 July 2011; published online: 22 August 2011.

Citation: Hughes SW, Lörincz ML, Blethyn $K$, Kékesi KA, Juhász G, Turmaine M, Parnavelas JG and Crunelli V (2011) Thalamic gap junctions control local neuronal synchrony and influence macroscopic oscillation amplitude during EEG alpha rhythms. Front. Psychology 2:193. doi: 10.3389/fpsyg.2011.00193

This article was submitted to Frontiers in Perception Science, a specialty of Frontiers in Psychology.

Copyright $\odot 2011$ Hughes, Lörincz, Blethyn, Kékesi, Juhász, Turmaine, Parnavelas and Crunelli. This is an open-access article subject to a non-exclusive license between the authors and Frontiers Media SA, which permits use, distribution and reproduction in other forums, provided the original authors and source are credited and other Frontiers conditions are complied with. 